\title{
INFLUENCE OF MAGNETIC IMPURITIES UPON UNIVERSAL CONDUCTANCE FLUCTUATIONS
}

\author{
T. DietL \\ Institute of Physics and College of Science, Polish Academy of Sciences \\ Al. Lotników 32/46, 02-668 Warszawa, Poland
}

\begin{abstract}
Mesoscopic phenomena in quantum structures which incorporate magnetic impurities with localized spins may exhibit a number of novel features driven by spin-disorder scattering, exchange spin-splitting of electron bands, and the formation of bound magnetic polarons. After brief information on these effects, their influence on universal conductance fluctuations as well as on low frequency noise and quantum localization is presented. Millikelvin investigations of diffusive charge transport, which have been carried out for submicron wires of $n^{+}-\mathrm{Cd}_{1-x} \mathrm{Mn}_{x}$ Te epilayers, are reviewed in some details. These studies have provided information on the significance of spin-disorder scattering in semiconductors and put into the evidence a new driving mechanism of the magnetoconductance fluctuations - the redistribution of the electrons between energy levels of the system, induced by the giant $s-d$ exchange spin-splitting. Important implications of these findings for previous interpretations of spin effects in semiconductor and metal nanostructures are discussed.
\end{abstract}

PACS numbers: 72.15.Rn, 73.20.Fz, 73.61.Ga, 75.50.Pp

\section{Introduction}

A considerable interest in transport properties of nanostructures containing localized spins was initially stimulated by theoretical predictions implying an unusual sensitivity of the conductance to the disorder distribution in a given mesoscopic sample [1]. This sensitivity to the potential realization results from the important role played in the diffusion by quantum interference of the de Broglie waves. It has been suggested [1] that even a single spin-flip of a localized spin might affect the conductance of the itinerant electrons. This would offer a powerful tool to probe dynamics of slow processes specific, in particular, to the spin-glass phase.

In this paper, we discuss selected theoretical developments and experimental findings concerning the influence of magnetic impurities with localized spins upon quantum transport phenomena in metals and doped semiconductors. These two groups of conductors form, in a sense, complementary systems. In particular, characteristic electronic lengths are typically shorter in metals than in semiconductors. By contrast, the range of magnetic inter-ion interactions are longer in 
metals than in semiconductors. Accordingly, in the case of semiconductors the subsystem of magnetic ions is more robust to the Kondo effect, spin-glass freezing, and the nanostructurization procedure. Another important aspect of diluted magnetic semiconductors is that the incorporation of magnetic impurities can be controlled during the growth process, while the details of the electronic structure, together with the value of the exchange coupling between the $d$ and $s$ electrons, are known precisely from quantitative studies of magnetooptic effects. The effective mass electrons in semiconductors are, however, often close to the localization boundary, unless a special doping procedure results in the reduction of strong electron scattering by ionized impurities.

\section{Universal conductance fluctuations, quantum noise, and quantum localization}

A convenient approach to the quantum effects is offered by the LandauerBüttiker formalism [2], according to which the process of electron transport can be viewed as a scattering problem of fluxes of the Fermi-level electrons entering and leaving the medium through ideal leads. Within this formalism the conductance can be expressed by eigenvalues of the familiar quantum-mechanical transmission and reflection matrices for the corresponding electron fluxes. These matrices contain information on the potential distribution in a given sample and are subject to symmetry constraints imposed, for instance, by spin degeneracy occurring if no spin-dependent perturbations are present. Without too much simplification, all novel quantum phenomena in question are caused by two effects, which to a large extent determine the spectrum of the eigenvalues. The first is quantum interference of transition amplitudes corresponding to different electron paths through the medium. The second is a certain spectral rigidity of the eigenvalues, which can be traced back to the celebrated Mott observation that in disordered systems states close in energy are far apart in a real space. Similarly to the case of a picture produced by interference of light, a change of the conductance occurs over a scale corresponding to the length of the interfering waves. Such an effect was observed as irregular but reproducible conductance fluctuations as a function of the gate voltage, i.e., the de Broglie wavelength of the electrons at the Fermi level $[3,4]$. Since the magnetic field (vector potential) affects also the phase of the wave function, similar aperiodic fluctuations occur as a function of the magnetic field $[3,4]$, the correlation field corresponding to one flux through the sample surface $S, B_{c} \approx h / e S$. Of course, in multiconnected geometries, a substantial number of electron trajectories encompasses the same magnetic flux, and the fluctuations acquire a periodic component, an expectation confirmed in a series of beautiful experiments with rings $[3,4]$. An interesting aspect of the conductance fluctuations is the universality of their magnitude, closely related to the spectral rigidity mentioned above. According to diagrammatic calculations [5,6] and confirmed by numerical simulations $[6,7]$, the root mean square (rms) value of the fluctuation amplitude is given by $\operatorname{rms}(\delta G) \approx 0.5 e^{2} / h$ in the case of the two-terminal conductance $G$ of any metallic sample at zero temperature.

Since the conductance contains information on the actual impurity distribution in a given sample, a question arises as to how much $G$ changes on average 
upon displacing a single scattering center, say, by $\delta R[8,1]$. The ratio of $\operatorname{rms}\left(G_{1}\right)$ to $\operatorname{rms}(\delta G)$ given above is evaluated to be for $d=3$ (cf. $[8,1]$ )

$$
\operatorname{rms}\left(G_{1}\right) / \mathrm{rms}(\delta G) \approx\left[C\left(\delta R k_{\mathrm{F}} \sigma_{\mathrm{imp}} / L \ell\right]^{1 / 2},\right.
$$

where $C(x \rightarrow 0)=x^{2} / 12$ and $C(x \rightarrow \infty)=1 ; \ell=\tau v_{\mathrm{F}}$ is the electron mean-free path while $\sigma_{\mathrm{i}} \mathrm{mp}=1 / n_{\mathrm{imp}} \tau_{\mathrm{imp}} v_{\mathrm{F}}$ is the cross-section of the relevant impurity, which in the case of, for example, neutral centers in semiconductors is substantially smaller than that corresponding to the unitary limit, $\sigma_{\mathrm{imp}} \approx 1 / k_{\mathrm{F}}^{d-1}$. (In the case of $d=2$ there is no $L$ in the denominator of Eq. (1).) The above result means that the mean response of the conductance to an uncorrelated displacement of a fraction $p$ of all impurities in the sample, $\operatorname{rms}\left(\delta G_{p}\right)=\left(p L^{d} n_{\text {imp }}\right)^{1 / 2} \mathrm{rms}\left(\delta G_{1}\right)$, grows with the system size $L$ (this will continue until $\mathrm{rms}\left(\delta G_{p}\right)$ reaches the saturation value $\operatorname{rms}(\delta G)$ ). A strong sensitivity of $G$ on the movement of impurities has led to the suggestion that the quantum effects in question are responsible for $1 / f$ noise $[8,9]$, a conjecture corroborated by its sensitivity to the magnetic field $[8,9]$. Clearly, the incoherent processes mentioned above (atom diffusion, but also spin fluctuations or vertex motion, etc.) operate only at nonzero temperature, at which one should also take into consideration temporal fluctuations of the potential, caused by phonons and/or other electrons. With the increasing temperature, the amplitude of such fluctuations grows, and so does $\operatorname{rms}\left(\delta G_{p}(L)\right)$, reaching finally its saturation value $\operatorname{rms}(\delta G)$. The size of the sample is then equal to the so-called phase-breaking length $L_{\varphi}$. At still higher temperatures the sample can be considered as consisting of $\left[L / L_{\varphi}(T)\right]^{d}$ uncorrelated "coherence boxes". In this regime, the classical self-averaging property of $G$ is recovered as the sensitivity of $G$ to, say, a magnetic field decreases with $L$ and increases with $L_{\varphi}$. In addition to $L_{\varphi}(T)$, there exists a one more relevant length scale at nonzero temperatures, which is associated with an average of the interference effects by thermal spread of the de Broglie wavelengths $\Delta \lambda$ of electrons contributing to $G$. This spread is of significance once $\Delta \lambda / \lambda^{2}$ becomes greater than the inverse of a mean length of electron path through the sample, i.e. if $L>L_{T}=\sqrt{\hbar D / k_{\mathrm{B}} T}$. Explicit expressions describing the decay of the universal conductance fluctuations (UCF) and quantum noise with $L$ for $L>L_{\varphi}, L_{T}$ can be found in [3,8,5-6]. In semiconductors, below $4 \mathrm{~K}$, usually $L_{\varphi}>L_{T}>\ell$.

The above discussion might suggest that the quantum effects are only important in mesoscopic samples $L_{\varphi}, L_{T}>L>\ell$, while they become totally insignificant at $L \gg L_{\varphi}, L_{T}$. Actually there are two quantum phenomena which survive the averaging procedure and contribute to the conductance of disordered macroscopic samples. Interestingly enough, they are thought to drive Anderson-Mott localization in disordered conductors [10]. The first is quantum interference corresponding to the clockwise and counterclockwise electron trajectories along the same self-intersecting electron paths. Since such interference is constructive, it increases the probability of return to the starting point, and thus diminishes the conductivity. The second effect stems from the earlier mentioned disorder-induced correlation between electron energies and their position in real space. Such an additional correlation introduces modifications to the standard Landau theory of Fermi liquids - the effects of the electron-electron interactions cannot be longer 
taken into account by a qualitatively unimportant renormalization of the effective mass. These two quantum effects turn out to be controlled by diffusion poles in the particle-particle and particle-hole correlation functions of the Fermi liquid in the random potential.

\section{Influence of magnetic impurities upon quantum transport}

Soon after the discovery that the Anderson-Mott localization in disordered metals and the universal conductance fluctuations in mesoscopic conductors are controlled by interference phenomena, i.e., diffusion poles in particle-particle and particle-hole correlation functions, it has become clear [5, 1, 11-12] that the exchange coupling between the carriers and the subsystem of magnetic impurities, $-J s S$, would play an important role in the physics of quantum transport. Indeed, it is well established by now that the localized spins, apart from introducing an additional temperature and magnetic-field dependent contribution to the momentum relaxation rate, $1 / \tau_{s}(T, H)$, can affect quantum transport phenomena in many other ways, depending on their dynamics, ordering, and relevant degrees of freedom. We discuss separately effects of spin-disorder scattering, spin-splitting, and magnetic polarons.

\subsection{Spin-disorder scattering}

In a spin-glass phase, the perturbing potential associated with the frozen spins leads to the violation of the Onsager-Büttiker symmetry relations in mesoscopic samples [13-15] as well as it alters the scaling factor of the UCF amplitude $[5,7]$. The fluctuating spins, in turn, because of an extreme sensitivity of the conductance to potential realizations, are expected to be an efficient source of the conductance noise $[7-8,1,16]$. If, therefore, the integration time of the resistance meter is longer than the correlation time of the spin fluctuations and $\tau_{s}$ is shorter than characteristic times of competing phase breaking mechanisms, the exchange interaction results in dumping of the UCF amplitude [17-19]. Besides, electron scattering by disordered Ising spins gives rise to a cutoff in the particle-particle channels with $j_{z}=0$ and the particle-hole channels with $j_{z}= \pm 1$, whereas the coupling to Heisenberg spins introduces a cutoff $1 / \tau_{s}$ to all diffusion poles except for the particle-hole channel with total spin $j=0$. This affects the quantum corrections to the conductivity, and alters the universality class of the metal-to-insulator transition (MIT) [10].

Quantitatively, in the presence of a magnetic field, the relevant longitudinal and transverse spin relaxation rate of electron liquid with a three-dimensional (3D) density of states assumes a well-known form [20]

$$
\frac{1}{\tau_{s \|, \perp}}=\frac{k_{\mathrm{B}} T \alpha^{2} m^{*} k_{\mathrm{F}}}{4 \pi \hbar^{3} g^{2} \mu_{\mathrm{B}}^{2}} \chi_{\|, \perp}(T, H),
$$

where $\alpha$ is the $s-d$ exchange integral; $\chi_{\|}(T, H)=\partial M(T, H) / \partial H$ and $\chi_{\perp}(T, H)=$ $M(T, H) / H$. Here $M(T, H)$ is the magnetization of the Mn spins, which is usually well parameterized by a modified Brillouin function, $M(T, H)=\bar{x} N_{0} g \mu_{\mathrm{B}} S B_{S}(T+$ $T_{0}, H$ ), where $\bar{x} N_{0}$ and $T+T_{0}$ are an effective concentration and temperature of the localized spins, respectively. 
In the vicinity of the MIT, the spin-spin correlation function of the electron liquid becomes significantly renormalized by disorder and electron-electron interactions [21]. In particular, the apparent spin relaxation rate $T_{2}$, as measured by linewidth of electron spin-resonance (ESR) or spin-flip Raman scattering (SFRS), is given by [22]

$$
\frac{1}{T_{2}}=\left(\frac{2}{\tau_{s \|}}+\frac{2}{\tau_{s \perp}}\right)\left[1+\frac{3 \sqrt{3}}{\pi\left(k_{\mathrm{F}} \ell\right)^{2}}\right]\left(1+\frac{F}{2}\right),
$$

where $\ell$ is the mean free path and $F$ is an amplitude of the Coulomb interaction [10].

\subsection{Spin-splitting}

The $s-d$ coupling results in the spin-splitting of the electron states that depends on the temperature and the magnetic field according to [20]

$$
\hbar \omega_{s} \equiv \tilde{g} \mu_{\mathrm{B}} H=g^{*} \mu_{\mathrm{B}} H+\alpha M(T, H) /\left(g \mu_{\mathrm{B}}\right) \text {. }
$$

As shown in Fig. 1 for electrons in $\mathrm{Cd}_{0.95} \mathrm{Mn}_{0.05} \mathrm{Se}$ : In [22], the spin-splitting energy can indeed be large at sufficiently low temperature, corresponding to the effective Landé factor in the excess of 500 .

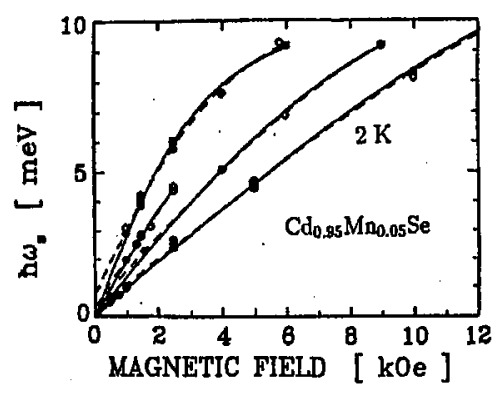

Fig. 1. Temperature dependence of the Stokes energy shift versus applied magnetic field obtained using spin-flip Raman scattering from itinerant electrons in $n^{+}-\mathrm{Cd}_{0.95} \mathrm{Mn}_{0.05} \mathrm{Se}$ in the vicinity of the metal-to-insulator transition. The open circles are for forward scattering, the solid circle - for back scattering. The solid lines show fits to Eq. (4), the dotted lines take into account a field independent bound magnetic polaron energy (after [22]).

Such spin-splitting alters the universality class of the MIT [10] and the scaling factor of the UCF amplitude [5] as it introduces a cutoff in the particle-hole channels with $j_{z}= \pm 1$ and particle-particle channels with $j_{z}=0[10,23-24]$. This leads also to a giant positive magnetoresistance in the neighborhood of the MIT $[23,25]$. At the same time, the spin-splitting leads to a redistribution of the carriers between the spin subbands. It was so far noted that the redistribution may affect the conductivity in two ways. First, by its influence on the mean free path that appears for $\hbar \omega_{s} \geq \varepsilon_{\mathrm{F}}$ [26]. Second, by changing the distance of $\varepsilon_{\mathrm{F}}$ to the mobility edge $\varepsilon_{\mathrm{m}}[27]$, an effect operating if $\hbar \omega_{s} \geq\left|\varepsilon_{\mathrm{F}}-\varepsilon_{\mathrm{m}}\right|$. 
Experimental results [28], which are discussed below, show that in mesoscopic systems the redistribution of the carriers between the nanostructure energy levels constitutes a new driving mechanisms of UCF in the magnetic field.This is because the redistribution and the corresponding shift of the Fermi energy $\varepsilon_{F}$ with respect to the bottom of the spin-down and spin-up subbands, result in a gradual change of energy levels of the system, which contribute to the conductance. The effect begins to show up at rather small values of the spin-splitting, $\hbar \omega_{s} \geq \max \left(k_{\mathrm{B}} T, h / T_{2}\right)$.

In order to evaluate the correlation field $H_{c}^{d-1}$ of those magnetoconductance fluctuations that are induced by the spin-splitting, $H_{c}^{\text {spin }}$, we observe that as long as $\varepsilon_{\mathrm{F}} \gg \hbar \omega_{\mathrm{s}}$, an increase in the magnetic field by $\Delta H$ leads to a shift of the Fermi energy $\Delta \varepsilon_{\mathrm{F}}= \pm \frac{1}{2} \Delta H \partial \hbar \omega_{s} / \partial H$. Hence, for $\hbar \omega_{s}>\mu_{\mathrm{c}}$, where [5] $\mu_{\mathrm{c}} \approx$ $\max \left(k_{\mathrm{B}} T, h / \tau_{\varphi}\right)$ is the energy correlation range, we obtain $H_{\mathrm{c}}^{\text {spin }}$ in the form

$$
H_{\mathrm{c}}^{\mathrm{spin}}=\sqrt{2} \mu_{\mathrm{c}}\left(\partial \mathrm{\hbar} \omega_{\mathrm{s}} / \partial H\right)^{-1},
$$

where the factor $1 / \sqrt{2}$ appears because the fluctuations result from a superposition of two independent contributions associated with two different spin subbands. It can be presumed that in magnetic materials phase breaking time $\tau_{\varphi} \equiv h / \mu_{\mathrm{c}}$ is equivalent to the spin-relaxation time $T_{2}$ of itinerant electrons. Since the correlation field for the orbital effects $[5,3]$

$$
H_{\mathrm{c}}^{\mathrm{orb}} \approx \frac{h / e}{L_{\min }^{(x)} L_{\min }^{(y)}},
$$

where $L_{\min }^{(i)}=\min \left(L_{\varphi}, L^{(i)}\right)$ and $L^{(x)} L^{(y)}$ is the sample area projected perpendicular to $H$, we see that the spin effect will dominate for sufficiently large values of the magnetic susceptibility $\chi(T, H)=\partial M(T, H) / \partial H$ and the inverse diffusion constant $D^{-1}=3 m^{*} /\left(\hbar k_{\mathrm{F}} \ell\right)$.

\subsection{Bound magnetic polarons}

There is a growing amount of evidences in favor of a phenomenological two-fluid model of electronic states in the vicinity of the MIT $[29,23,22,30]$. According to that model the conversion of itinerant electrons into local moments occurs gradually, and begins already on the metal side of the MIT, leading to the coexistence of the extended and strongly localized states. In magnetic materials, the local moments can polarized, via $s-d$ interaction, the neighboring $\mathrm{Mn}$ spins (there are about $100 \mathrm{Mn}$ ions within the Bohr orbit in $n$-Cd $\mathrm{Cd}_{0.99} \mathrm{Mn}_{0.01} \mathrm{Te}$ ). The ferromagnetic bubbles (bound magnetic polarons) formed in this way constitute centers of spin-dependent scattering for itinerant electrons. The efficiency of this scattering increases rather steeply with decreasing temperature, as the degree of the bubble polarization is proportional to the magnetic susceptibility of $\mathrm{Mn}$ spins, $\chi(T)[20,23]$.

\section{Experimental results for submicron wires of $\mathrm{Cd}_{1-x} \mathrm{Mn}_{x}$ Te epilayers}

$\mathrm{Cd}_{x} \mathrm{Mn}_{1-x} \mathrm{Te}:$ In films with $x=0$ or $x=1 \pm 0.1 \%$, a typical thickness of $0.3 \mu \mathrm{m}$, and electron concentrations around $10^{18} \mathrm{~cm}^{-3}$ were grown by MBE onto (001) oriented SI GaAs epiready substrates with $10 \AA \mathrm{ZnTe}$ and $3 \mu \mathrm{m} \mathrm{CdTe}$ undoped buffer layers $[31,28]$. The electron concentration in the studied samples 
is by a factor of five greater than that corresponding to the MIT. The carrier and $\mathrm{Mn}$ concentrations are low enough to preclude an appearance of either spin-glass freezing or the Kondo effect, even at the lowest temperature of interest here, $T=$ $30 \mathrm{mK}[32]$.

The studied wires had the form of six-terminal Hall bars with a square cross-section $S$ of a side $W=0.3 \pm 0.05 \mu \mathrm{m}$, and the distance between the voltage probes being $4-5 \mu \mathrm{m}$, as shown in the inset to Fig. 2. They were fabricated by means of $30 \mathrm{keV}$ electron-beam lithography, followed by wet etching. Low-frequency a.c. currents down to $100 \mathrm{pA}$ were employed for the resistance measurements in a dilution refrigerator, carefully protected against electromagnetic noise.

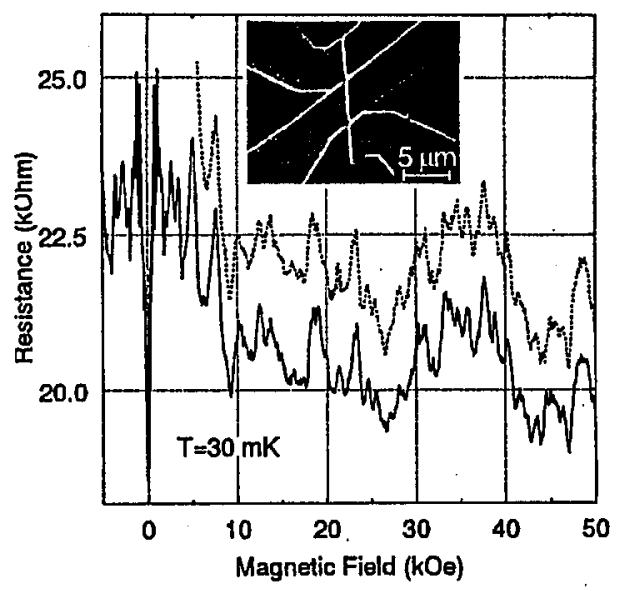

Fig. 2. Two measurements of the resistance as a function of the magnetic field for the wire of $n^{+}-\mathrm{Cd}_{0.99} \mathrm{Mn}_{0.01} \mathrm{Te}$ at $30 \mathrm{mK}$. The inset shows a scanning electron micrograph of the sample (after [28]).

Figures 2 and 3 present resistance as a function of the magnetic field perpendicular to wires of $\mathrm{CdTe}: \mathrm{In}$ and $\mathrm{Cd}_{0.99} \mathrm{Mn}_{0.01} \mathrm{Te}: \mathrm{In}$ with the electron concentration $1.0 \times 10^{18}$ and $8 \times 10^{17} \mathrm{~cm}^{-3}$, respectively [28]. Weak-field magnetoresistance and irregular reproducible resistance fluctuations are detected in both materials. Starting from the magnetoresistance we note that because down to $100 \mathrm{mK}, W$ is greater than the thermal diffusion length $L_{T}=\sqrt{\hbar D / k_{\mathrm{B}} T}$, the studied wires are three dimensional (3D) in respect to phenomena that are sensitive to thermal broadening of the distribution function, such as electron-electron interactions. Since, however, in nonmagnetic wires $L_{\varphi}=\sqrt{D \tau_{\varphi}}>L_{T}$ [23], we may expect a dimensional crossover in the negative magnetoresistance as it is controlled by phase breaking effects. This dimensional crossover is shown in Fig. 3a, which displays the temperature dependence of the magnetoresistance $\Delta \rho$ in $\mathrm{n}^{+}-\mathrm{CdTe}$. The theoretical curves were calculated for the $3 \mathrm{D}$ case from the weak-localization theory [10], taking $m^{*} / m_{0}=0.099$ and assuming $L_{\varphi}=A / T^{3 / 4}$, where $A$ was a fitting parameter determined to be $0.9 \mu \mathrm{m} \mathrm{K}^{3 / 4}$. Since in 3D $\Delta \rho \propto H^{-1 / 2}$, while in 1D 

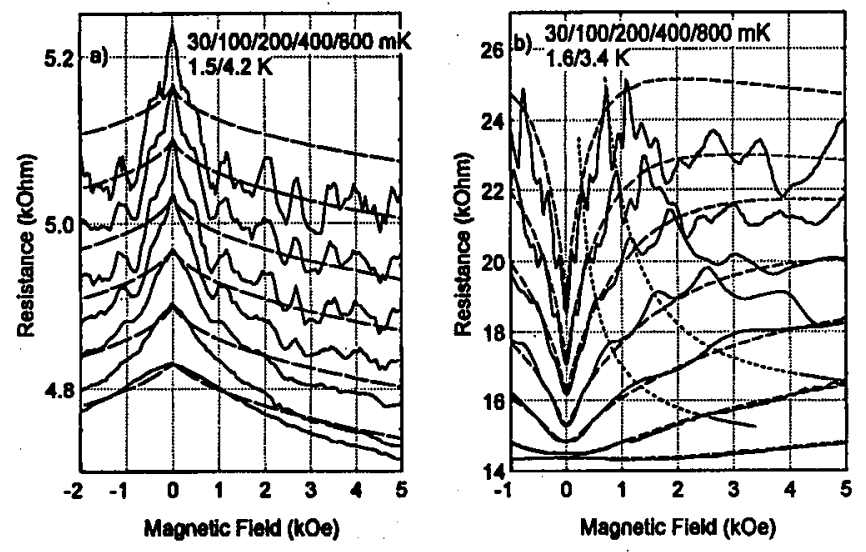

Fig. 3. Resistance changes as a function of the magnetic field for the wires of $n^{+}-\mathrm{Cd}_{1-x} \mathrm{Mn}_{x} \mathrm{Te}$ with $x=0$ (a) and $x=1 \%$ (b) at various temperatures between $30 \mathrm{mK}$ and $4.2 \mathrm{~K}$ (traces for the lowest temperatures are shifted upwards). Dashed lines represent magnetoresistance calculated in the framework of $3 \mathrm{D}$ weak-localization theory $[10,23]$. Dotted lines are guides for the eye, and visualize a strong temperature dependence of the resistance features in $\mathrm{Cd}_{0.99} \mathrm{Mn}_{0.01} \mathrm{Te}$ (b) (after [28]).

$\Delta \rho \propto H^{-1}$, the discrepancies between the experimental and calculated $\Delta \rho$, appearing at $L_{\varphi}(T)>W$, seem to indicate the presence of the temperature induced crossover from $3 \mathrm{D}$ to $1 \mathrm{D}$ at about $3 \mathrm{~K}$ in the studied wire.

A striking influence of the magnetic impurities upon the magnetoresistance and UCF is shown in Fig. $3 \mathrm{~b}$, where data for $\mathrm{n}^{+}-\mathrm{Cd}_{0.99} \mathrm{Mn}_{0.01} \mathrm{Te}$ are shown together with the results of a theoretical computation $[10,23]$. As demonstrated previously [23], the positive magnetoresistance is caused by the effect of the giant exchange spin-splitting upon the electron-electron interaction. By taking the well-known material parameters of $\mathrm{Cd}_{1-x} \mathrm{Mn}_{x} \mathrm{Te}[20,33] \alpha N_{0}=0.22 \mathrm{eV}, N_{0}=$ $1.48 \times 10^{22} \mathrm{~cm}^{-3}, g=2.0, S=5 / 2$ and, for $\bar{x}=1 \%, T_{0} \approx 80 \mathrm{mK}[32,33]$ one obtains $\hbar \omega_{s}(T, H)$ that gives $\tilde{g} \mu_{\mathrm{B}} H=5.5 \mathrm{meV}$ for $S g \mu_{\mathrm{B}} H \gg k_{\mathrm{B}}\left(T+T_{0}\right)$ and $\tilde{g}=150 \mathrm{~K} /\left(T+T_{0}\right)$ in the opposite limit. In that way, the Coulomb amplitude was the only adjustable parameter and its fitting yielded $g_{3}+g_{4}=1.3$ [28]. As expected, no dimensional crossover to $1 \mathrm{D}$ is observed in the positive magnetoresistance as it is controlled by a short length scale, $L_{T}$.

Turning to the resistance fluctuations in the studied samples we note that their root mean square amplitude is independent of the magnetic field. On the other hand, it increases with decreasing temperature according to $\operatorname{rms}(\Delta R) / R^{2} \approx$ $(C / T)^{r} e^{2} / h$ where, above $100 \mathrm{mK}, C=0.1 \mathrm{mK}$ and $r=0.5$. While such a behavior is typical for nonmagnetic $1 \mathrm{D}$ wires, in which the distance between the voltage probes is greater than both $L_{T}$ and $L_{\varphi}[5,3]$, it came as a surprise in the case of $\mathrm{Cd}_{0.99} \mathrm{Mn}_{0.01} \mathrm{Te}$. Indeed, in the latter $\mathrm{rms}(\Delta R)$ is expected to be controlled by $\tau_{s}$ which for $T>T_{0}$ is independent of temperature but increases with the magnetic field [17-19]. However, because of the low density of states specific to semiconductors, one obtains $\hbar /\left(k_{\mathrm{B}} \tau_{s}\right)=\alpha^{2} m^{*} k_{\mathrm{F}} \bar{x} N_{0} S(S+1) /\left(4 \pi \hbar^{2} k_{\mathrm{B}}\right)$ to be 
as low as $100 \mathrm{mK}$ at $H=0$. This estimate explains the minor importance of spin-disorder scattering in the studied system.

Another important aspect of the data depicted in Fig. $3 \mathrm{~b}$ concerns an unusual behavior of the correlation field $H_{\mathrm{c}}$ of the resistance fluctuations in $\mathrm{Cd}_{0.99} \mathrm{Mn}_{0.01} \mathrm{Te}$. As shown by dotted lines, the fields $H_{i}$ corresponding to characteristic points of the fluctuation pattern tend to increase with either temperature or the magnetic field, a behavior not observed in nonmagnetic wires, including those of $n^{+}-\mathrm{CdTe}$. This new effect is visible not only for the perpendicular but also for the parallel orientation of the magnetic field with respect to the wires.

We note that the field-induced changes of spin configurations have been proposed as the mechanism driving magnetoconductance fluctuations in spin-glass $\mathrm{Cu}: \mathrm{Mn}$ wires [15]. However, it has recently been demonstrated [28] that the dominant mechanism has its origin in the spin-splitting-induced redistribution of the carriers between the spin subbands, an effect that can also operate in the paramagnetic phase considered here, as discussed in the previous section. Since, according to Eq. (4), the spin-splitting is proportional to the magnetization, the positions of the characteristic points of the fluctuation pattern should be temperature independent if the resistance would be plotted as a function of the magnetization $M$, not of the magnetic field $H$. That this is indeed the case is shown in Fig. 4 . Moreover, by putting parameters suitable for the $\mathrm{CdMnTe}$ wire $\left(k_{\mathrm{F}} \ell=1.5\right.$ and $F=2), H_{\mathrm{c}}^{\text {spin }}=410 T[\mathrm{~K}]$ Oe can be obtained from Eqs. (5) and (6). Except for the lowest temperatures, where the effect of bound magnetic polaron may appear $[23,22]$, this agrees with the experimental values, $H_{c}^{\exp }=360 T[\mathrm{~K}]+36 \mathrm{Oe}$, determined in the range $0.03 \mathrm{~K} \leq t \leq 0.8 \mathrm{~K}$ and $0 \leq H \leq k_{\mathrm{B}}\left(T+T_{0}\right) /\left(g \mu_{\mathrm{B}}\right)$.

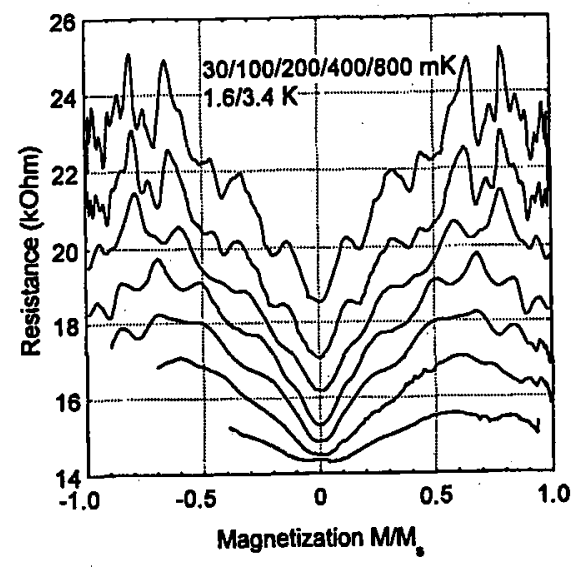

Fig. 4. Magnetoresistance data of Fig. $3 \mathrm{~b}$ plotted as a function of magnetization in the units of $M_{s}=g \mu_{\mathrm{B}} S N_{0} \tilde{x}$ (after [28]). 


\section{Discussion and conclusions}

The experimental results presented above reemphasize a relatively small importance of spin-disorder scattering in semiconductors. This, in particular, put into a question a suggestion [19] that spin-disorder scattering by etching=induced defects can account for the absence of the resistance fluctuations in the region of weak magnetic fields in $\mathrm{n}^{+}$-GaAs wires at $T \geq 1.3 \mathrm{~K}$.

The data for CdMnTe demonstrate unambiguously the significant influence of the spin-splitting-induced redistribution of the carriers between the spin subbands upon transport phenomena of mesoscopic systems. It is interesting to find out whether the spin-splitting effect in question could account for the finite value of $H_{\mathrm{c}}^{\text {spin }}$ observed in $\mathrm{Cu}: \mathrm{Mn}$ [15]. By taking parameters suitable for 1000-atomic-ppm $\mathrm{Cu}: \mathrm{Mn}$ [15], i.e., $\ell=200 \AA, L_{\varphi}=0.35 \mu \mathrm{m}, m^{*}=m_{0}, \chi=\chi_{C}\left(T_{\mathrm{g}}\right)=$ $3.8 \times 10^{-4} \mathrm{emu}$, and $\left|\alpha N_{0}\right|=0.5 \mathrm{eV}$, one obtains [28] $H_{\mathrm{c}}^{\text {spin }}=4.1 \mathrm{kOe}$ from Eqs. (5) and (6), a value quite close to the experimental finding, $H_{\mathrm{c}}^{\text {spin }}=4.2 \div 6.4 \mathrm{kOe}$ [15].

Finally, we note that further experimental effort is needed to confirm the assignment of the increase in noise [16] and the breakdown of the Onsager-Büttiker relations $[14,15]$ in $\mathrm{Cu}: \mathrm{Mn}$ to the spin-glass freezing.

This work was supported by the Committee for Scientific Research, grant No. 2PO3B 06411.

\section{References}

[1] B.L. Altshuler, B.Z. Spivak, Pis'ma Zh. Eksp. Teor. Fiz. 42, 363 (1985) [JETP Lett. 42, 477 (1986)]; S. Feng, A.J. Bray, P.A. Lee, M.A. Moore, Phys. Rev. B 36, 5624 (1987).

[2] R. Landauer, IBM J. Res. Dev. 32, 306 (1988); M. Büttiker, ibid. 32, 317 (1988).

[3] Mesoscopic Phenomena in Solids, Eds. B.L. Altshuler, P.A. Lee, R.A. Webb, Elsevier, Amsterdam 1991; S. Washburn, R.A. Webb, Rep. Prog. Phys. 55, 1311 (1992).

[4] C.V.J. Beenakker, H. Van Houten, Solid State Phys. 44, 1 (1991).

[5] B.L. Altshuler, B.I. Shklovskii, Zh. Eksp. Teor. Fiz. 91, 220 (1986) [Sov. Phys., JETP 64, 127 (1986)].

[6] P.A. Lee, A.D. Stone, H. Fukuyama, Phys. Rev. B 35, 1039 (1987).

[7] M. Cieplak, B. Bułka, T. Dietl, Phys. Rev. B 44, 12337 (1991); ibid. 51, 8939 (1995).

[8] S. Feng, in: Mesoscopic Phenomena in Solids, Eds. B.L. Altshuler, P.A. Lee, R.A. Webb, Elsevier, Amsterdam 1991, p. 107.

[9] N. Giordano, in: Mesoscopic Phenomena in Solids, Eds. B.L. Altshuler, P.A. Lee, R.A. Webb, Elsevier, Amsterdam 1991, p. 131.

[10] B.L. Altshuler, A.G. Aronov, in: Electron-Electron Interaction in Disordered Systems, Eds. A.L. Efros, M. Pollak, North-Holland, Amsterdam 1985, p. 1; H. Fukuyama, ibid. p. 155; P.A. Lee, T.V. Ramakrishnan, Rev. Mod. Phys. 57, 287 (1985); D. Belitz, T.R. Kirkpatrick, ibid. 66, 261 (1994).

[11] P.A. Lee, J. Non-Cryst. Solids 35/36, 21 (1980); S. Hikami, A.I. Larkin, Y. Nagaoka, Prog. Theor. Phys. 63, 707 (1980).

[12] G. Bergmann, Phys. Rep. 107, 1 (1984).

[13] S. Hershfield, Phys. Rev. B 44, 3320 (1991); J. Liu, M.A. Pennington, N. Giordano, Phys. Rev. B 45, 1267 (1992). 
[14] P.G.N. de Vegvar, L.P. Levy, T.A. Fulton, Phys. Rev. Lett. 66, 2380 (1991); M.B. Weissman, ibid. 68, 3468 (1992); P.G.N. de Vegvar, L.P. Lévy, ibid. 68, 3485 (1992).

[15] P.G.N. de Vegvar, T.A. Fulton, Phys. Rev. Lett. 71, 3537 (1993).

[16] N.E. Israeloff, M.B. Weissman, G.J. Nieuwenhuys, J. Kasiorowska, Phys. Rev. Lett. 63, 794 (1989); N.E. Israeloft, G.B. Alers, M.B. Weissman, Phys. Rev. B 44, 12613 (1991).

[17] A. Benoit, S. Washburn, R.A. Webb, D. Mailly, L. Dumoulin, in: Anderson Localization, Eds. T. Ando, H. Fukuyama, Springer, Berlin 1988, p. 346; C. Van Haesendonck, H. Vloeberghs, Y. Bruynseraede, R. Jonckheere, in: Nanostructures Physics and Fabrication, Eds. M.A. Reed, W.P. Kirk, Academic Press, Boston 1989, p. 467; A. Benoit, D. Mailly, P. Perrier, P. Nedellec, Superlattices Microstruct. 11, 313 (1992); T. Dietl, G. Grabecki, J. Jaroszyński, Semicond. Sci. Technol. 8, S141 (1993); G. Grabecki, T. Dietl, W. Plesiewicz, A. Lenard, T. Skośkiewicz, E. Kamińska, A. Piotrowska, Physica B 194-196, 1107 (1994).

[18] A.A. Bobkov, V.I. Falko, D.E. Khmelnitskii, Zh. Eksp. Teor. Fiz. 98, 703 (1990) [Sov. Phys., JETP 71, 393 (1990)]; V. Chandrasekhar, P. Santhanam, D.E. Prober, Phys. Rev. B 42, 6823 (1990); V.I. Falko, J. Phys., Condens. Matter 4, 3943 (1992).

[19] A.K. Geim, S.V. Dubonos, I.Yu. Antonova, Pis'ma Zh. Eksp. Teor. Fiz. 52, 873 (1990) [JETP Lett. 52, 247 (1990)].

[20] T. Dietl, in: Handbook on Semiconductors, Ed. T.S. Moss, Vol. 3b, North-Holland, Amsterdam 1994, p. 1251.

[21] S. Sachdev, Phys. Rev. B 34, 6049 (1986).

[22] T. Dietl, M. Sawicki, E.D. Isaacs, M. Dahl, D. Heiman, M.J. Graf, S.I. Gubariev, D.L. Alov, Phys. Rev. B 43, 3154 (1991).

[23] M. Sawicki, T. Dietl, J. Kossut, J. Igalson, T. Wojtowicz, W. Plesiewicz, Phys. Rev. Lett. 56, 508 (1986).

[24] Y. Ono, J. Kossut, J. Phys. Soc. Jpn. 53, 1128 (1984).

[25] Y. Shapira, N.F. Oliveira Jr., P. Becla, T.Q. Vu, Phys. Rev. B 41, 5931 (1990).

[26] Y. Shapira, R.L. Kautz, Phys. Rev. B 10, 4781 (1974).

[27] H. Fukuyama, Y. Yosida, Physica $B+C .105,132$ (1981); T. Wojtowicz, T. Dietl, M. Sawicki, W. Plesiewicz, J. Jaroszyński, Phys. Rev. Lett. 56, 2419 (1986); B.A. Aronzon, N.K. Chumakov, T. Dietl, J. Wróbel, Zh. Eksp. Teor. Fiz. 105, 405 (1994) [Sov. Phys. JETP 78, 216 (1994)].

[28] J. Jaroszyński, J. Wróbel, M. Sawicki, E. Kamińska, T. Skośkiewicz, G. Karczewski, T. Wojtowicz, A. Piotrowska, J. Kossut, T. Dietl, Phys. Rev. Lett. 75, 3170 (1995).

[29] R.N. Bhatt, M.A. Paalanen, S. Sachdev, J. Phys. (France) 49, C8-1179 (1988).

[30] P. Głód, T. Dietl, T. Fromherz, G. Bauer, I. Miotkowski, Phys. Rev. B 49, 7797 (1994).

[31] G. Karczewski, A. Zakrzewski, M. Kutrowski, J. Jaroszyński, W. Dobrowolski, E. Grodzicka, E. Janik, T. Wojtowicz, J. Kossut, Acta Phys. Pol. A 87, 241 (1995).

[32] M.A. Novak, O.G. Symko, D.J. Zheng, S. Oseroff, J. Appl. Phys. 57, 3418 (1985).

[33] J.A. Gaj, W. Grieshaber, C. Bondin-Deshayes, J. Cibert, G. Feuillet, Y. Merle d'Aubigné, A. Wasiela, Phys. Rev. B 50, 5512 (1994). 